\title{
Resistencia al fuego de pórticos simples de acero a dos aguas
}

\section{Fire resistance of single pitched-roof steel portal frame}

\author{
J. J. Ferrán Gozálvez $^{(*)}$, C. Ferrer Gisbert ${ }^{(*)}$, M. Redón Santafé ${ }^{(*)}$, F. J. Sánchez Romero ${ }^{(*)}$, J. B. Torregrosa Soler ${ }^{(*)}$
}

\section{RESUMEN}

El método habitual de análisis en situación de incendio consiste en el cálculo simplificado de barras aisladas. Es conservador al aplicarse a estructuras con capacidad de redistribuir esfuerzos. Se desconoce el tipo de fallo que afecta a la seguridad de las personas y extensión del fuego.

Se utiliza el análisis avanzado para obtener el comportamiento a fuego de un pórtico simple a dos aguas de acero conforme a la normativa española.

Se establece una metodología mediante un programa de cálculo estructural de propósito general (SAP200o), con un análisis dinámico no lineal del material (plástico) y geométrico (P-Delta y grandes desplazamientos), y propiedades mecánicas y térmicas dependientes de la temperatura. Se analizan los diferentes tipos de colapso. Se efectúan estudios paramétricos sobre la influencia de la rigidez de la base, protección de los soportes y cargas actuantes. Este cálculo puede permitir justificar mayores tiempos de resistencia al fuego que el análisis simplificado de barras.

Palabras clave: seguridad a fuego; análisis por elementos finitos; naves agroindustriales; cálculo plástico.

\section{ABSTRACT}

The standard procedure of structural fire design is based on the simplified analysis of single members. This method leads to conservative results in the case of structures able to redistribution of forces. The failure mechanism affecting both life safety and fire propagation is unknown.

This work proposes a methodology for the advanced fire calculation of single pitched-roof portal frame for an agroindustrial building according to the Spanish Specifications with the structural software SAP2OOO.

A non-linear dynamic and plastic, geometric (P-Delta and large-displacements) calculation method has been developed. The different failure mechanisms and their influence are studied in terms of fire time resistance, human hazard and good safety. Also, parametric analyses were conducted: load level, rotational stiffness of the base and finally, support fire protection.

Keywords: fire safety; finite element analysis; agro-industrial buildings; plastic frame analysis.

(*) Universidad Politécnica de Valencia. DIRA (Valencia, España).

Persona de contacto/Corresponding author: jjferran@agf.upv.es (J. J. Ferrán Gozálvez)

ORCID: http://orcid.org/oooo-0002-0548-300o (J. J. Ferrán Gozálvez); http://orcid.org/oooo-0002-4287-5281

(C. Ferrer Gisbert); http://orcid.org/oooo-0002-4581-1590 (M. Redón Santafé); http://orcid.org/oooo-0003-1447-6897

(F. J. Sánchez Romero); http://orcid.org/oooo-0003-2052-5827 (J. B. Torregrosa Soler)

Cómo citar este artículo/Citation: Ferrán Gozálvez, J. J., Ferrer Gisbert, C., Redón Santafé, M., Sánchez Romero, F. J., Torregrosa Soler, J. B. (2016). Resistencia al fuego de pórticos simples de acero a dos aguas. Informes de la Construcción, 69(545): e172, doi: http:// dx.doi.org/10.3989/ic.15.089.

Copyright: (C) 2017 CSIC. Licencia / License: Salvo indicación contraria, todos los contenidos de la edición electrónica de Informes de la Construcción se distribuyen bajo una licencia de uso y distribución Creative Commons Attribution License (CC BY) Spain 3.o. 


\section{INTRODUCCIÓN}

El pórtico simple a dos aguas es la tipología estructural más habitual en las edificaciones agroindustriales. El acero expuesto al fuego es bastante vulnerable debido a su elevada conductividad térmica y a la rápida pérdida de resistencia y rigidez. En muchas situaciones es necesario proyectar una protección que retrase el incremento de temperatura del acero para que el edificio mantenga su capacidad portante el tiempo requerido. Se encarece la estructura resultando menos competitiva.

La verificación de la capacidad portante en caso de incendio se realiza habitualmente mediante el análisis simplificado de barras aisladas de la Instrucción de Acero Estructural (1) (EAE, art. 46). Se verifica que el efecto de la acción de incendio función del fuego de cálculo adoptado no debe ser mayor a la resistencia del elemento sometido a una distribución de temperaturas en cualquier instante del proceso de incendio.

En el Reglamento de seguridad contra incendios en los establecimientos industriales (RSCIEI) (2) se establecen los requisitos de estabilidad al fuego de la estructura de un edificio de carácter industrial. La estructura principal de cubiertas ligeras (peso inferior a $1 \mathrm{kN} / \mathrm{m}^{2}$ ), cuando su fallo no pueda ocasionar daños graves a establecimientos próximos, o bien no se exige resistencia al fuego (tipo $\mathrm{C}$ de bajo riesgo intrínseco) o bien se exigen resistencias de 15, 30 o 60 minutos de estabilidad mecánica, según el caso.

Mediante el método simplificado es frecuente que el tiempo resistido por pórticos a dos aguas de cubierta ligera con grados de utilización (relación entre el efecto de las acciones en situación de incendio y la resistencia del perfil en el instante inicial del incendio) entre 0,2 y 0,3, se sitúe entre 13 a 17 minutos. Para esta tipología estructural este resultado es conservador, lo que repercute desfavorablemente en condiciones de proyecto si se decide una protección del acero innecesaria o sobredimensionada.

Los métodos de cálculo estructural avanzado proporcionan resultados más realistas al evaluar la respuesta de la estructura con el incremento de temperatura. Se consideran la modificación de las propiedades mecánicas y térmicas del acero y el efecto no despreciable de las dilataciones.

Se puede justificar, hasta un cierto margen, un mayor tiempo de resistencia a fuego si existe capacidad de redistribuir esfuerzos. Otro aspecto de este tipo de análisis es que permite mejorar el diseño estructural a fuego para evitar formas de colapso inaceptables que puedan repercutir en la seguridad de las personas (evacuación y labores de extinción) y prevenir la extensión del fuego.
Los dinteles a dos aguas sometidos a cargas gravitatorias experimentan una inestabilidad geométrica global para una determinada flecha vertical en cumbrera. Si los apoyos extremos presentan un cierto grado de coacción al desplazamiento horizontal y giro (debido a su unión al pilar), es posible que los dinteles mantengan una posición de equilibrio estable en una posición invertida respecto a la inicial. En este trabajo se denomina STB (snap-through-buckling) al instante de pérdida de estabilidad (Figura 1). En ciertas circunstancias la estructura mantiene su capacidad portante un tiempo prolongado después del STB.

Los objetivos de este trabajo son:

1. Establecer un método de cálculo estructural avanzado a fuego mediante un programa informático de propósito general (SAP2000).

2. Definir y obtener la temperatura crítica a la que ocurre el colapso estructural conforme a las prescripciones de la normativa española.

3. Análisis de la influencia de los parámetros más determinantes: rigidez y capacidad resistente de la base del pórtico, base y pilares con o sin protección frente al fuego, nivel de cargas y otros.

\section{ANTECEDENTES}

Debido a que los edificios industriales en situación de incendio presentan diferencias en relación a las exigencias normativas y procedimientos constructivos de cada país, la difusión de los trabajos es limitada. Destacan Reino Unido, Australia y Nueva Zelanda, países en que se ha analizado con mayor rigor y profundidad el comportamiento del pórtico a dos aguas frente al fuego.

En estos países el cálculo plástico se utiliza de forma habitual y tradicional en el dimensionado de pórticos a dos aguas, especialmente de bases articuladas, razón por la que el cálculo estructural a fuego es más accesible. La publicación SCI P313 (3) aborda el análisis a fuego de estructuras aporticadas, de uno o varios vanos, con vigas de alma llena o en celosía, que delimitan un establecimiento o un sector de incendio.

La normativa del Reino Unido no requiere en pórticos de naves un tiempo de resistencia a fuego. Para evitar la extensión del fuego la separación entre edificios debe ser superior a $15 \mathrm{~m}$ o bien se debe evitar el colapso hacia el exterior del recinto. El SCI P313 (3) recomienda dimensionar la base de pilares y cimentación para soportar un momento de vuelco (OTM: overturning moment) para evitar el colapso hacia el exterior de los paramentos verticales. Se deducen expresiones simples muy utilizadas aunque las bases del método han sido cuestionadas.

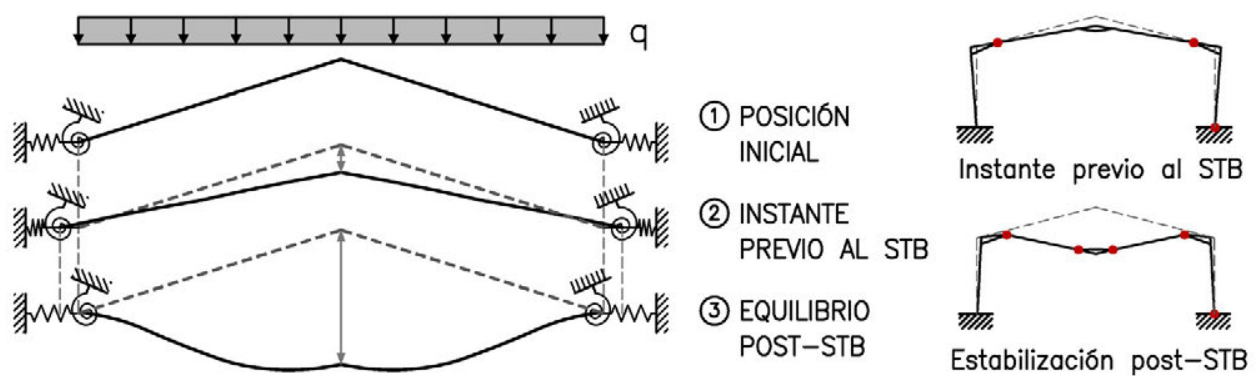

Figura 1. Pérdida transitoria de estabilidad en dinteles a dos aguas. 
Wong, Burguess y Plank (4), mediante cálculo plástico, obtuvieron sencillas expresiones para predecir la temperatura crítica de colapso hasta el inicio de la pérdida de estabilidad de los dinteles. Wong (5) realizó tres ensayos reales a fuego de un pórtico a dos aguas de $6 \mathrm{~m}$ de luz con perfiles doble $\mathrm{T}$ y bases articuladas. Desarrolló cálculos estáticos no lineales, elástico-plásticos, en dos dimensiones (2D) hasta el instante del STB.

Vassart et al. (6) llevaron a cabo análisis 2D y 3D no lineales, elastoplásticos, utilizando un método implícito dinámico para predecir el punto de re-estabilización de los dinteles de un pórtico doble a dos aguas. Dos equipos de trabajo emplearon el programa Abaqus, otro grupo el programa Ansys y el último el programa Safir. Los resultados mostraron curvas semejantes de evolución de los desplazamientos en cumbrera, esquina del pórtico y axil del dintel en función del tiempo, aunque las magnitudes presentaban cierta dispersión.

Bong (7) investigó con el programa Safir el comportamiento 2D y 3D de pórticos con diversas localizaciones y severidad del incendio, sin y con fase de decaimiento, rigidez de la base, contribución de correas, restricción aportada por el muro hastial, arriostramiento lateral de pilares, revestimiento con hormigón de pilares y conexión cerramiento-pilares.

Song (8) y Song et al. (9) continuaron el trabajo de Wong (5) con el programa Vulcan con un cálculo cuasi-estático-dinámico para predecir y analizar la re-estabilización del dintel de un pórtico simple.

Rahman et al. (10) ampliaron los estudios paramétricos utilizando el programa Abaqus en un pórtico simple, profundizando en el análisis de los momentos de vuelco en la base de pilares.

Moss et al. (11), en base a la normativa neozelandesa y procedimientos constructivos de ese país, realizan un exhaustivo análisis $3 \mathrm{D}$ para determinar el modo de colapso y tiempo de resistencia al fuego de pórticos a dos aguas.
El-Heweity (12) realizó un estudio numérico con Safir de un pórtico a dos aguas con perfil tubular cuadrado. Planteó varios escenarios y pendientes del dintel.

\section{MODELO NUMÉRICO}

Se utiliza el programa de elementos finitos SAP200o (13). Se modeliza el pórtico a dos aguas como estructura plana de barras que se encuentra debidamente arriostrada lateralmente a temperatura ambiente. Los nudos tienen impedido el desplazamiento según la dirección normal al plano de la estructura y los giros alrededor de los ejes contenidos en el plano de la estructura. Para ello contribuyen la rigidez aportada por el material de cubierta y fachada, su unión a las correas, de éstas con la estructura portante y los sistemas de arriostramiento de cubierta y fachada. Esta estabilidad se mantiene durante el incendio, lo cual puede no ocurrir con fuegos localizados intensos que ocasionen una destrucción importante de los paramentos y uniones.

En cambio, no se considera el beneficio que puedan aportar las correas en la re-estabilización del dintel por el efecto catenaria transversal al pórtico afectado. Este efecto es apreciable cuando el fuego actúa sobre un número reducido de pórticos. Las correas se debilitan antes por el fuego, pudiendo sufrir inestabilidad por la compresión al dilatar. También pueden debilitarse sus uniones a la chapa y estructura y comprometer su estabilidad a flexión. Posteriormente, las tracciones que por efecto catenaria re-estabilizan las correas y el dintel, deben ser soportadas por riostras y muros hastiales cuya capacidad puede ser insuficiente.

\subsection{Geometría}

Se establecen cuatro tipos de pórticos de diferentes características geométricas, perfiles y cargas (Figura 2). El acero es del tipo S275.

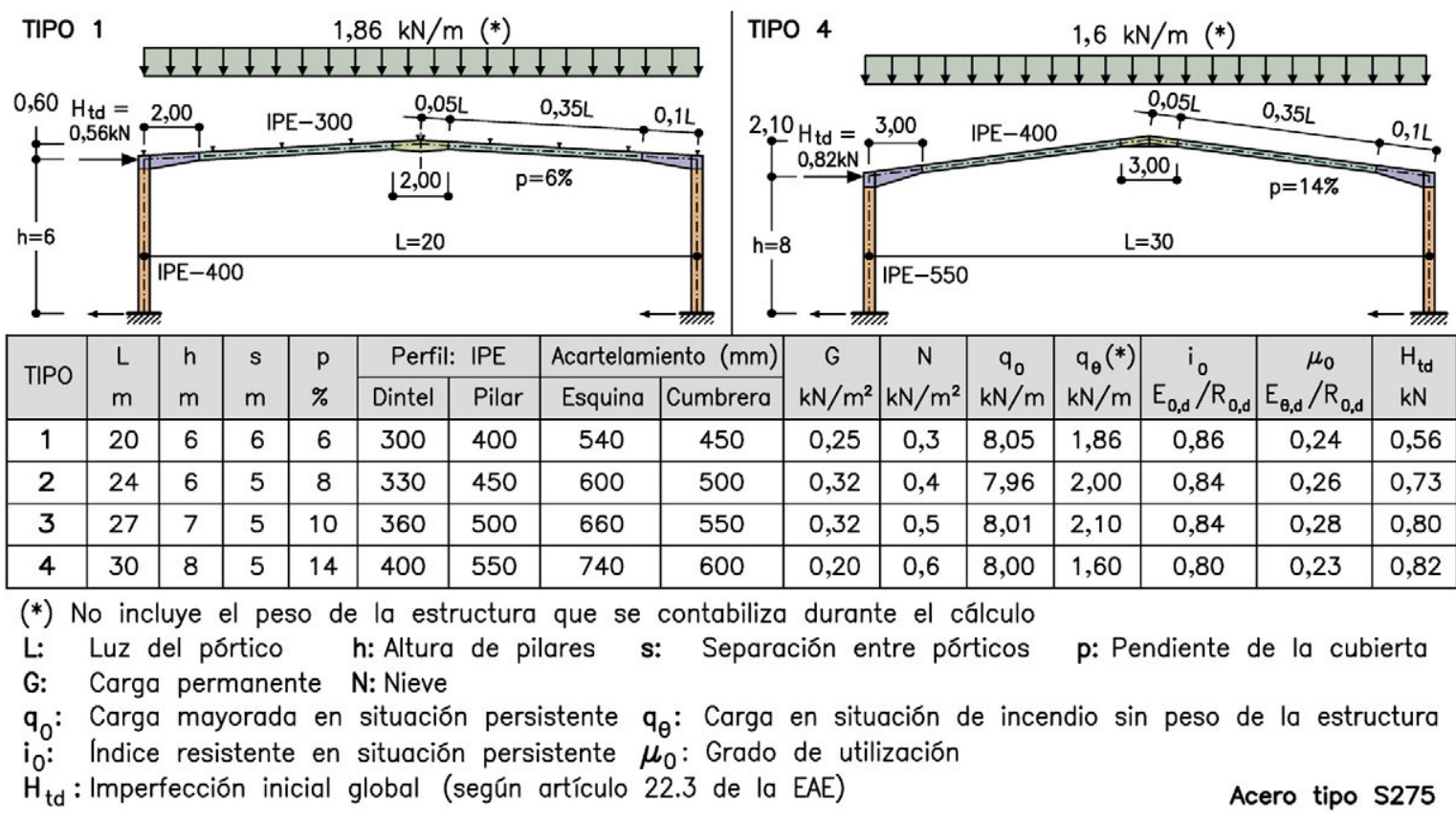

Figura 2. Características y variables de cálculo de los pórticos analizados. 
Se efectúa el dimensionado en estado límite último y situación persistente. El índice resistente $\mathrm{i}_{\mathrm{o}} \leq 1$ es la relación entre el efecto de las acciones y la resistencia a temperatura ambiente.

\subsection{Bases de cálculo}

Las acciones de cálculo en situación de incendio se obtienen de la combinación accidental en situación extraordinaria del Código Técnico, Seguridad Estructural (CTE-SE):

$$
\sum_{\mathrm{j} \geq 1} \mathrm{G}_{\mathrm{k}, \mathrm{j}}+\mathrm{A}_{\mathrm{d}}+\psi_{\mathrm{i}, \mathrm{1}} \cdot \mathrm{Q}_{\mathrm{k}, \mathrm{1}}+\sum_{1>1} \psi_{2, \mathrm{i}} \cdot \mathrm{Q}_{\mathrm{k}, \mathrm{i}}
$$

Donde $\mathrm{G}$ son las acciones permanentes, $\mathrm{A}_{\mathrm{d}}$ la acción térmica (dilataciones) y $\mathrm{Q}_{\mathrm{k} 1}$ es la acción variable determinante (nieve, $\Psi_{1.1}=0,2$ ). No se consideran, en cubiertas no accesibles, acciones variables acompañantes $\mathrm{Q}_{\mathrm{k}, \mathrm{i}}$ al $\operatorname{ser} \psi_{2 . \mathrm{i}}=\mathrm{O}$ para el viento y sobrecarga de uso.

En algún caso se considera el viento como acción variable determinante (el CTE-SE adopta $\psi_{1.1}=0,5$ frente al Eurocódigo y EAE que es $\left.\Psi_{1,1}=0,2\right)$.

\subsection{Bases de pilares}

El comportamiento de la base del pilar es un factor importante en relación al fuego:

\section{Rigidez}

En cada pórtico se consideran cuatro tipos de rigidez de la base: articulada, nominalmente articulada (rigidez $0,4 \mathrm{EI}_{\text {pilar }} / \mathrm{h}$ ), nominalmente rígida (rigidez $4 \mathrm{EI}_{\text {pilar }} / \mathrm{h}$ ) y empotrada, siendo $I_{\text {pilar }}$ la inercia del pilar y $h$ su altura. Se modelizan mediante un apoyo elástico de muelle a flexión (moment spring).

\section{Resistencia}

Se denomina capacidad resistente limitada de la base del pilar al caso en que la base de anclaje tenga menor resistencia que el perfil del pilar. La base de anclaje se suele dimensionar para resistir el flector en la base del pilar que en muchas ocasiones es inferior al flector máximo que solicita el pilar.

En los casos de base nominalmente rígida y empotrada puede ocurrir que no llegue a desarrollarse una rótula en la base del perfil del pilar si la resistencia de la base de anclaje es inferior al momento plástico del pilar. Se supone que una base de anclaje con pernos a tracción es capaz de desarrollar un comportamiento plástico, cuya resistencia es la máxima en ese punto.

En este caso la capacidad resistente y rigidez de la base se modeliza con un elemento de enlace de soporte sin longitud (link support) plástico con ciclo de histéresis según Wen (14).

\subsection{No linealidad del material}

El cálculo se realiza con fracciones de carga, discretamente crecientes, de la carga constante y uniforme en el dintel en situación de incendio (salvo niveles de carga cercanos a la formación de cada rótula, que el programa establece internamente), hasta alcanzarse la resistencia última de las secciones y, si es el caso, la formación del mecanismo de colapso. Se desconoce a priori el número, posición y longitud de las rótulas plásticas. Es necesario definir en las barras un determinado número de secciones susceptibles de plastificarse (plastic hinges). Para ello se divide cada pilar y dintel en 20 elementos, se define una posible rótula en cada sección intermedia y en los empotramientos, y dos más en cada nudo pertenecientes a los extremos de cada barra, contabilizándose 84 posiciones de posibles rótulas plásticas. Con ello se pretende captar la plastificación progresiva a lo largo de la longitud de la barra a elevadas temperaturas post-STB. La posición de las rótulas puede cambiar con la temperatura, revertir (descarga) y volver a formarse con esfuerzos y giro opuestos.

Las secciones de las rótulas son de Clase 1. Se desprecia la interacción axil-flector debido a que no es preciso reducir la resistencia plástica a flexión para las secciones en doble $\mathrm{T}$ utilizadas y para los pequeños axiles existentes en todo el proceso.

Para definir la relación Momento-Rotación en las rótulas plásticas se realizaron los siguientes cálculos previos:

1. Se considera el diagrama tensión-deformación (Figura 3) para métodos de cálculo avanzado y las propiedades del material dependientes de la temperatura que se obtienen mediante coeficientes correctores $\mathrm{K}_{\theta}$ del límite elástico $\left(\mathrm{f}_{\mathrm{y}, \theta}\right)$, límite de proporcionalidad $\left(\mathrm{f}_{\mathrm{p}, \theta}\right)$ y módulo de elasticidad del acero $\left(\mathrm{E}_{\theta}\right)$, según la EAE (1).

2. Se modeliza la sección en fibras plásticas, 40 (alas) y 20 (alma) (fiber hinge). Se obtienen las curvas Momento-Rotación para los pórticos definidos y para cada temperatura de la Figura 3.

3. Se establece un diagrama simplificado Momento-Rotación $(\mathrm{M} / \varphi)$ (Figura 4) para los cálculos de este trabajo.
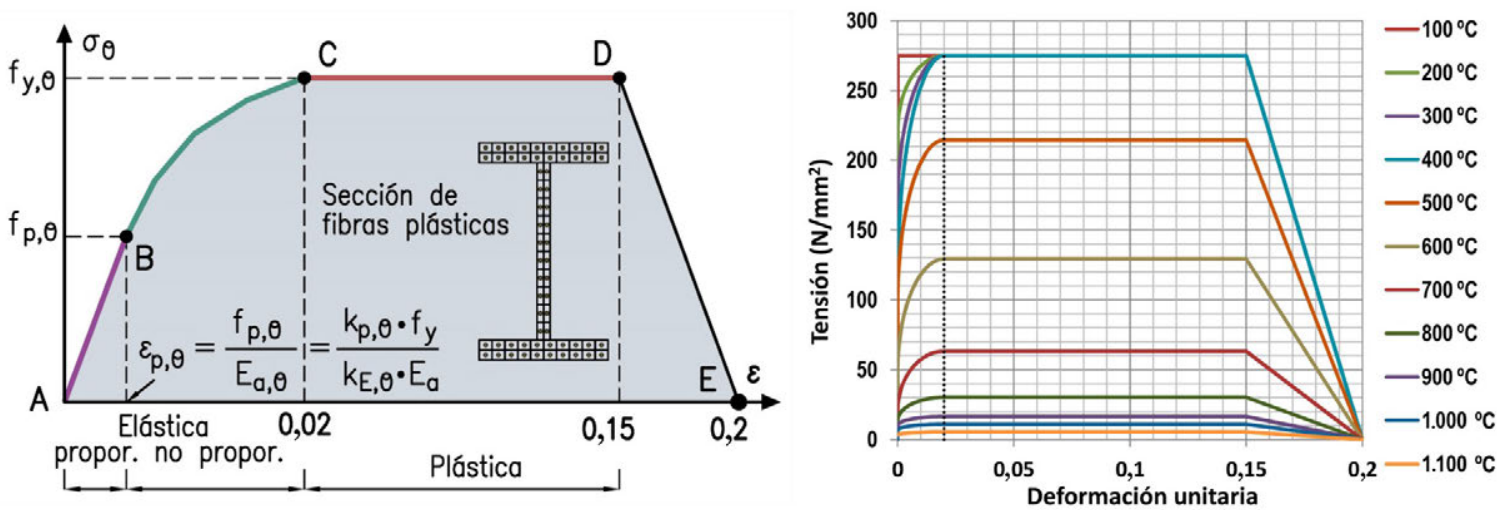

Figura 3. Curva tensión deformación del acero a elevada temperatura y variación de las curvas con la temperatura. 


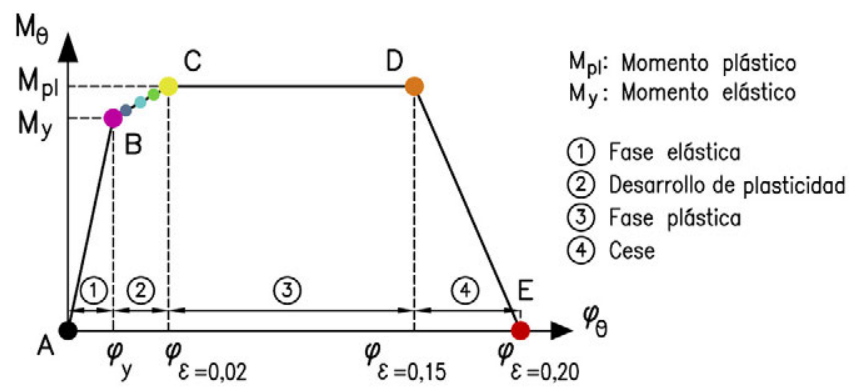

Figura 4. Diagrama Momento-Rotación simplificado adoptado en los cálculos.

\subsection{No linealidad geométrica}

Se activa mediante un cálculo P-Delta global, p-delta local en barra y en grandes desplazamientos (large displacements). Se formulan las ecuaciones de equilibrio en la posición deformada, considerando el cambio de posición y orientación de los elementos, ignorando los cambios de forma y tamaño.

Para generar una respuesta asimétrica que origine, si es el caso, diferencias apreciables del desplome entre ambos pilares, según se constata en incendios reales, se aplica una acción horizontal en cabeza del pilar de valor total $\mathrm{H}_{\mathrm{td}}$ (Figura 2), equilibrada en la base de los pilares con una fuerza total igual y de signo contrario.

Esta acción representa además las imperfecciones globales por defecto de verticalidad en elementos comprimidos. Se calcula según prescribe la EAE en el apartado 22.3.3. Para ello se han multiplicado los axiles de la combinación más desfavorable en situación persistente para cada pilar, por los coeficientes $\mathrm{k}_{\mathrm{h}} \mathrm{y} \mathrm{k}_{\mathrm{m}}$.

\subsection{Cálculo dinámico}

Es suficiente un cálculo estático para analizar la respuesta de la estructura hasta el instante previo al STB. El cálculo dinámico es un instrumento para obtener el comportamiento estructural posterior al STB.

Carece de interés precisar el comportamiento dinámico real posterior al STB. Consiste en una solución al problema de cálculo por falta de convergencia del método de Newton-Ralphson para pendientes negativas en la relación carga-deformación, debidas a esta inestabilidad transitoria.

Se realiza un análisis implícito dinámico no lineal mediante el método de la integración directa. Mediante el cálculo previo de los modos de vibración se estima un número de escalones de carga (steps) entre 1.200 a 2.000 , mayor a más temperatura. La carga se aplica gradualmente según una ley senoidal y se mantiene constante entre un 20 a $50 \%$ del tiempo de carga. El factor de amortiguamiento se aumenta del valor habitual de 0,02 a un valor entre 0,15 y 0,25. Mantener un tiempo la carga y aumentar el factor de amortiguamiento permiten minimizar las vibraciones transitorias que alteran las deformaciones y esfuerzos posteriores al STB.

\subsection{Comportamiento térmico de la sección}

El modelo de fuego es la curva normalizada tiempo-temperatura UNE-EN 1363 (Figura 5). No representa un fuego real (temperatura creciente sin fase de cese), aunque se utiliza ampliamente en el cálculo. Supone que se alcanza el estado de combustión generalizada (flashover), no habitual en recintos de gran volumen y altura como las naves, con posibilidad de ventilación por destrucción de lucernarios y paramentos.

Se considera una distribución homogénea de temperaturas en cada sección, hipótesis bastante aceptada para este tipo de construcciones.

En este trabajo se utilizan dos tipos de sección en relación a su comportamiento térmico que simulan las situaciones extremas:

- Sección sin protección: sometida totalmente al fuego normalizado UNE-EN 1363 (Tabla 48.1 de la EAE para una sección abierta totalmente expuesta al fuego). Para obtener la temperatura homogénea de la sección en función del tiempo en minutos de la curva normalizada tiempo-temperatura (Figura 5) se aplica la ecuación en diferencias finitas del artículo 48 de la EAE (1), sobre el perímetro envolvente de la sección aplicando el factor de sombra $\mathrm{k}_{\mathrm{sh}}<1$.

- Sección protegida: se mantiene a temperatura ambiente $\left(20^{\circ} \mathrm{C}\right)$ durante todo el tiempo de exposición al fuego.

Las situaciones intermedias no analizadas en este trabajo (exposición a tres caras o hasta la mitad del perímetro según el art. 48.1 de la EAE, con revestimientos protectores según el art. 48.2 de la EAE) proporcionarían resultados entre ambas situaciones.

Se adoptan las expresiones del artículo 45.1 de la EAE para el coeficiente de dilatación lineal y calor específico, variables con la temperatura.

\subsection{Escenarios de incendio}

Durante el desarrollo de un incendio el aire más caliente se expande y acumula bajo la cubierta. Como elemento no protegido el dintel se considera sometido a la acción del fuego con una temperatura uniforme en toda su longitud en todos los casos analizados.

En muchos incendios de naves la temperatura del aire desciende con la altura. En la base, corrientes de aire más frescas alimentan al fuego, especialmente si ocurre una destrucción de lucernarios o existen aberturas de ventilación en la cubierta. En muchos casos una cara del soporte es exterior o puede

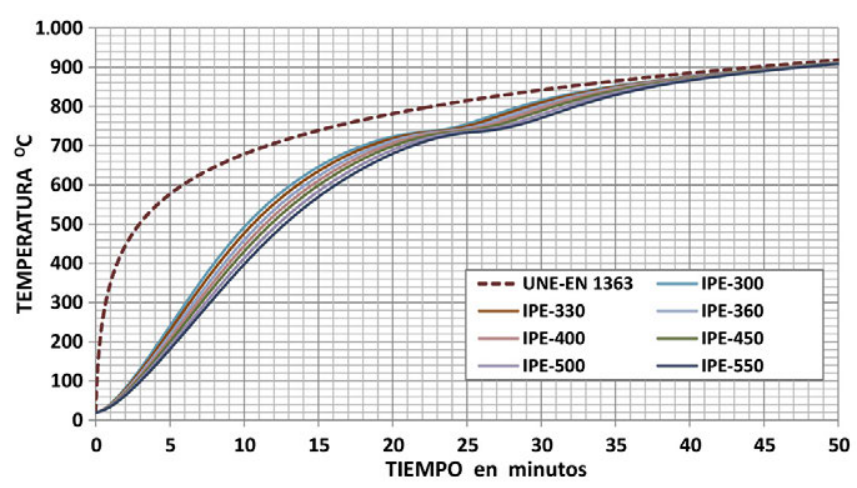

Figura 5. Curva de temperatura de fuego normalizado UNE-EN 1363 y de los perfiles de los pórticos analizados. 
estar parcial o totalmente protegida por los elementos constructivos de la fachada.

En otras situaciones menos habituales el pilar resulta afectado por el fuego en toda su altura, en cuyo caso se puede considerar un escenario de fuego generalizado que afecta por igual a toda la estructura.

Respecto a la base de anclaje del pilar se formula la hipótesis de base afectada por el fuego (se asignó idéntica temperatura a la del pilar) o base a temperatura ambiente (protegida). En la práctica habitual la base de anclaje suele disponerse bajo la solera, embebida en el hormigón. Además, debido a su gran masividad y al reducido contacto con los pernos, hace asumible en muchos casos esta última hipótesis de funcionamiento.

Se han definido tres situaciones:

- Pórtico y bases sin proteger.

- Pórtico sin proteger y base protegida.

- Soporte y base protegidas.

En todos los casos se consideró un escenario que afecta simétricamente a los dos pilares y dinteles.

\subsection{Análisis estructural}

El modelo de análisis estructural dinámico no lineal del material y geométrico se implementa para cada temperatura hasta que se alcanza la temperatura de ruina o fin de cálculo, con variación de las propiedades mecánicas y térmicas del material con la temperatura.

La carga gravitatoria es constante en todo el proceso térmico. La carga térmica (dilatación) depende de la temperatura del acero.

Para cada parámetro analizado (Tabla 1) se obtiene una curva que relaciona deformación o esfuerzo con la temperatura. La curva se traza a partir de entre 100 y 150 puntos. Los puntos singulares (formación de rótulas, punto STB, ruina o fin de cálculo) se obtienen con precisión de $1^{\circ} \mathrm{C}$.

\subsection{Temperatura crítica y colapso estructural}

La temperatura crítica $\theta_{\text {a,cr }}$ es aquella tal que al ser alcanzada por el dintel se considera que el pórtico queda fuera de servicio. En los pórticos analizados el pilar es un perfil superior al dintel, por ello está a menor temperatura o a temperatura ambiente si está protegido.

No es posible establecer un criterio único objetivo para definir el instante de ruina estructural en situación de incendio. $\mathrm{Su}$ influencia en el cálculo del tiempo de resistencia al fuego es notable. La ruina, que queda a juicio del calculista y que depende de las condiciones de contorno, puede ocurrir en los siguientes escenarios:

- La magnitud del desplome de los pilares hacia el exterior es incompatible con la capacidad de deformación de los paramentos verticales, con riesgo de su derrumbe. Se suele considerar un colapso inaceptable.

Respecto al desplome de pilares compatible con los paramentos, la ruina dependerá del tipo de paramento, altura, tipología (voladizo, biapoyado). Se apuntan algunos valores límites del desplome ( $h$ : altura del pilar):

- El SCI P313 (3) adopta en general una inclinación de los soportes de $1^{\circ}(h / 57)$. Muchos autores estiman que es un valor conservador.

- Algunos textos aconsejan un límite de $h / 30$.

- En la normativa australiana (Australian Building Codes Board, 2004) para paneles prefabricados verticales de más de $5 \mathrm{~m}$ de altura se establece $\mathrm{He} / 25$, siendo $\mathrm{H}_{\mathrm{e}}$ la altura de la viga superior de sujeción del panel.

- Instantes después del STB se observa debilidad en la estructura por resultar un período muy corto de equilibrio y con deformaciones rápidamente crecientes.

- Posteriormente al STB cuando el desplome hacia el interior de la nave es inadmisible para los paramentos. Se trata de un modo de colapso aceptable.

- La flecha del dintel alcanza el nivel de la solera aunque los desplomes sean elevados.

- La deformación longitudinal unitaria del acero alcanza el valor $\varepsilon=0,2$.

- Tras la recuperación del equilibrio post-STB la estructura vuelve a experimentar otra fase de inestabilidad que provoca su colapso. En este caso el programa de cálculo no converge a una solución.

\subsection{Aportaciones respecto a estudios precedentes}

Se resumen los siguientes aspectos diferenciales de este trabajo frente a investigaciones anteriores:

- Se siguen las prescripciones de la EAE (cap. XII). Las condiciones de seguridad y acciones corresponden al Código Técnico de la Edificación: CTE SE y CTE SE-AE, respectivamente. En los estudios citados sólo se consideran las acciones permanentes.

Por ello se considera $1 / 5$ de la carga de nieve $\left(\psi_{1,1}=0,2\right)$. En algún caso se toma la mitad de la acción del viento $\left(\psi_{1,1}=0,5\right)$. Según el Eurocódigo, si se adopta $\psi_{2,1}$ para la acción variable principal (situación cuasipermanente), entonces la nieve y viento son nulas, lo que coindice con la normativa de muchos países.

- La plasticidad se define mediante la inserción en las secciones de las barras de posibles rótulas plásticas (hinges)

Tabla 1. Parámetros analizados más destacables.

\begin{tabular}{|l|l|}
\hline \multicolumn{1}{|c|}{ Parámetro analizado } & \multicolumn{1}{c|}{ Variación de los parámetros } \\
\hline Pórtico sin proteger. Base protegida & Articulado, nominalmente articulado, nominalmente rígido, empotrado \\
\hline Pórtico y base sin proteger & Nominalmente articulado, nominalmente rígido y empotrado \\
\hline Soportes y base protegidas & $\begin{array}{l}\text { Articulado, nominalmente articulado, nominalmente rígido, empotrado, capa- } \\
\text { cidad resistente limitada de la base del pilar }\end{array}$ \\
\hline Nivel de cargas & $0,6,0,8,1,1,2,1,4$ de la carga en situación de incendio \\
\hline $\begin{array}{l}\text { Longitud y canto del acartelamiento } \\
\text { en esquina }\end{array}$ & $\begin{array}{l}\mathrm{O}, 15,0,2,0,25,0,3 \text { fracción de la longitud del dintel } \\
1,1,1,2 \text { factor del canto del acartelamiento }\end{array}$ \\
\hline
\end{tabular}


cuyas propiedades son función de la temperatura. Este modelo representa más estrechamente el análisis plástico estructural, del cual se han deducido las expresiones de cálculo a fuego de algunas normativas.

- Se consideran las propiedades mecánicas reales del perfil que incluyen el acuerdo ala-alma y la variación precisa de las propiedades mecánicas en los acartelamientos.

- La capacidad resistente y rigidez de la base se modeliza mediante un elemento de enlace de soporte, más preciso y de uso más cómodo que los modelos estructurales que añaden barras ficticias para modelizar ambos parámetros.

- El límite elástico efectivo se adopta a la deformación del $2 \%$ (o,5\% según la normativa inglesa) y se considera la rama decreciente entre 15 y $20 \%$ (en los estudios citados no se acota la resistencia, que es ilimitada, con la deformación plástica).

- Para el cálculo de la temperatura del perfil se considera el perímetro envolvente de la sección en doble T en lugar del perímetro del perfil y se adopta el factor de sombra (que en otros estudios se omite siendo $\mathrm{k}_{\mathrm{sh}}=1$ ).

- Se considera el caso de bases protegidas y estructura sin proteger. En los estudios precedentes las bases se consideran a idéntica temperatura al soporte.

\section{ANÁLISIS DE RESULTADOS}

Los resultados obtenidos se presentan en forma de curvas que relacionan esfuerzo o deformación con la temperatura. En cada curva se anotan las temperaturas de puntos singulares: formación de rótulas plásticas, instante de inicio del STB y ruina o fin del cálculo. Los resultados obtenidos se comparan con los resultados de temperatura crítica del dintel calculados según el método simplificado del artículo 46.6 de la EAE para elementos aislados sometidos a compresión y flexión.
La causa de la ruina se indica mediante la siguiente nomenclatura:

S: La flecha vertical desciende al nivel de la solera.

D: La rótula alcanza el giro para la deformación longitudinal de $\varepsilon=0,2$.

Er: Se denomina «fin del cálculo» a la imposibilidad de que el programa encuentre una solución por problemas de convergencia en un tiempo razonable, por interrupción de la ejecución o bien los resultados son incompatibles con las condiciones de deformación y equilibrio. Puede ser debido a:

- La estructura es nuevamente inestable y colapsa.

- Por problemas de cálculo como, por ejemplo, que los parámetros de control del cálculo son inadecuados, incapacidad del método para encontrar una solución o errores internos.

Si se establece la ruina por desplome excesivo:

DE: Hacia el exterior. Es un colapso que puede considerarse inaceptable.

DI: Hacia el interior. Colapso aceptable.

Además se refiere como:

V: Los pilares mantienen su posición vertical. Situación óptima.

La Figura 6 representa la secuencia del proceso de formación de rótulas plásticas, instante del STB y ruina de dos de los casos analizados. El pórtico tipo 4 con soporte sin proteger y base empotrada protegida puede alcanzar la ruina por un

PÓRTICO TIPO 4 empotrado: Soporte sin proteger. Base protegida.

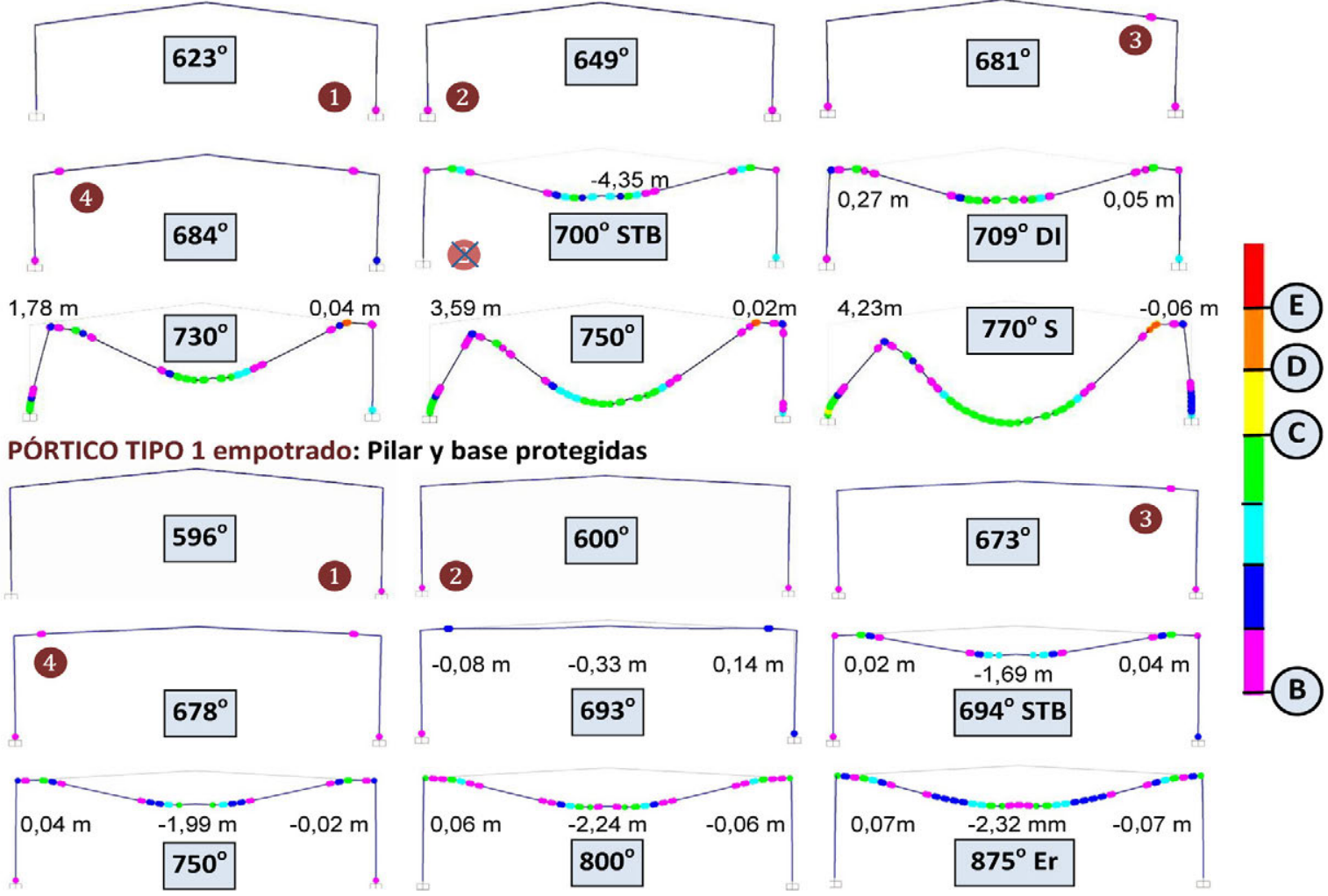

Figura 6. Deformada a escala, posición y evolución de rótulas plásticas del pórtico tipo 4 y tipo 1, según el código de colores y letras de la Figura 4. 
desplome excesivo, por ejemplo de $h / 30$, hacia el interior del pilar izquierdo, lo que ocurre a $709^{\circ} \mathrm{C}$. Si no es el caso, el pórtico alcanza el nivel del suelo a $770^{\circ} \mathrm{C}$. El pilar derecho se mantiene casi vertical.

El pórtico tipo 1 con pilares protegidos (Figura 6) tiene una secuencia similar de formación de rótulas plásticas. Si bien la protección de pilares tiene poca incidencia sobre el STB, el período post-STB es mucho más largo y los desplomes son más bajos. La máxima temperatura corresponde a fin del cálculo (Er).

En la Tabla 2 se ordenan las temperaturas y tiempos de resistencia al fuego de los casos analizados más representativos.

En las Figuras 7 y 8 se representan gráficamente la variación con la temperatura de la flecha vertical, el desplome de la esquina izquierda y esquina derecha, el flector en la base del pilar izquierdo y los axiles en el extremo inferior del dintel izquierdo con la temperatura.

Los pórticos analizados presentan similar respuesta de esfuerzos y deformaciones en función de la temperatura. El mecanismo previo al STB es de tipo combinado (pórticoviga), con rótulas plásticas en las bases de uno o ambos soportes y extremos de cartabones de esquina. En la fase post-STB se desarrollan y avanzan plastificaciones desde los extremos de los cartabones de cumbrera y desde la base de los pilares.

En todos los análisis realizados para el pórtico biarticulado se desarrolló el mecanismo de colapso o ruina definitiva en el instante del STB (Tabla 2). El pilar derecho se desploma en exceso hacia el exterior. Según el RSCIEI, para cualquier edificio tipo $\mathrm{C}$, si su derrumbe puede afectar a otros colindantes aunque la separación supere $3 \mathrm{~m}$, requiere una resistencia al fuego mínima R3o. Ello obliga a proteger si es el caso toda la estructura, pues proteger sólo el soporte se comprobó en los cálculos que no incrementa la temperatura crítica. Al considerar la carga de viento como acción variable determinante, incluso se obtuvieron temperaturas críticas más bajas (Tabla 2).

Las posiciones de las rótulas plásticas previas al STB de la Figura 6 son las habituales en los casos analizados, en igual o menor número, o en unos pocos casos, en otro orden de aparición o posición (en cabeza del pilar). Las rótulas pueden distribuirse en diferentes posiciones de las curvas de temperatura (Figura 7). Sin embargo, no se evidencia que afecten a la forma de las curvas ni a las temperaturas críticas.

Es necesaria una pequeña rigidez de la base (nominalmente articulada) para conseguir que las curvas se encuentren muy próximas a la curva de base empotrada, con diferencias de temperatura muy pequeñas en el instante del STB. Se debe garantizar la resistencia al flector en la base del pilar, pues se podría producir un desplome excesivo hacia el exterior.

Excepto para el pórtico de base articulada, el tramo post-STB es:

- Pórtico y bases sin proteger: muy pequeño o inapreciable. La ruina coincide con el instante previo al STB.

- Pórtico sin proteger y base protegida: se aprecia un tramo inclinado hasta un punto en que las deformaciones vuelven a crecer rápidamente. El tiempo en el instante del STB se sitúa entre 18' a 22'. El fin de cálculo tiene lugar entre 25' a 30'. Los desplomes son elevados.

- Pilares protegidos: se ha superado ampliamente en todos los casos los 30' de resistencia al fuego ya que el tramo post-STB es prolongado, superando los $850^{\circ} \mathrm{C}$ en todos los casos. Se comprobó que, incluso si se protege sólo 2/3 de altura del pilar desde la base, se superan los 30'.

- Pilares protegidos con base de capacidad resistente limitada: se acotó el flector en la base del pilar al obtenido a

Tabla 2. Temperaturas y tiempos de resistencia al fuego en el instante del STB y ruina, tipo de ruina para algunos de los casos analizados más relevantes de la Tabla 1. SP: sin protección de pilar y dintel, base protegida. Se sombrean los casos de la Figura 6.

\begin{tabular}{|c|c|c|c|c|c|c|c|c|c|c|c|}
\hline \multirow{3}{*}{ Bases del pilar } & \multirow{3}{*}{ Situación de diseño } & \multicolumn{5}{|c|}{ Tipo 1} & \multicolumn{5}{|c|}{ Tipo 4} \\
\hline & & \multicolumn{2}{|c|}{ STB } & \multicolumn{3}{|c|}{ Ruina } & \multicolumn{2}{|c|}{ STB } & \multicolumn{3}{|c|}{ Ruina } \\
\hline & & ${ }^{\circ} \mathbf{C}$ & mín & ${ }^{\circ} \mathbf{C}$ & mín & Tipo & ${ }^{\circ} \mathbf{C}$ & mín & ${ }^{\circ} \mathbf{C}$ & mín & Tipo \\
\hline \multirow{2}{*}{ Articuladas } & Pilar y base (indistinto: sin/con protección) & 677 & 16,53 & 677 & 16,53 & $\mathrm{D}, \mathrm{DE}$ & 674 & 17,92 & 674 & 17,92 & $\mathrm{D}, \mathrm{DE}$ \\
\hline & Viento (acción variable determinante) & 666 & 15,95 & 666 & 15,95 & $\mathrm{D}, \mathrm{DE}$ & 677 & 18,08 & 677 & 18,08 & $\mathrm{D}, \mathrm{DE}$ \\
\hline \multirow{2}{*}{ Nom. articuladas } & Sin protección (SP) & 689 & 17,25 & 760 & 25,40 & S, DI & 692 & 19,00 & 757 & 26,88 & S, DI \\
\hline & Pilar y base protegidos & 689 & 17,25 & 849 & 34,55 & Er, V & 698 & 19,42 & 794 & 29,82 & Er, V \\
\hline \multirow{2}{*}{ Nom. rígidas } & Sin protección (SP) & 689 & 17,25 & 760 & 25,40 & S, DI & 699 & 19,48 & 764 & 27,47 & S, DI \\
\hline & Pilar y base protegidos & 693 & 17,50 & 871 & 38,92 & Er, V & 772 & 28,10 & 873 & 40,13 & Er, V \\
\hline \multirow{6}{*}{ Empotradas } & Sin protección (SP) & 689 & 17,25 & 760 & 25,40 & $\mathrm{D}, \mathrm{DI}$ & 700 & 19,55 & 770 & 27,95 & S, DI \\
\hline & Pilar y base protegidos & 694 & 17,57 & 875 & 39,85 & Er, V & 775 & 28,33 & 876 & 40,78 & Er, V \\
\hline & o,8 factor de carga (SP) & 723 & 20,00 & 794 & 28,08 & $\mathrm{D}, \mathrm{DI}$ & 748 & 26,00 & 810 & 31,18 & S, DI \\
\hline & o,6 factor de carga (SP) & 769 & 26,12 & 841 & 33,25 & $\mathrm{D}, \mathrm{DI}$ & 787 & 29,25 & 846 & 35,28 & S, DI \\
\hline & o,3 fracción longitud del cartabón (SP) & 704 & 18,27 & 770 & 26,20 & $\mathrm{D}, \mathrm{DI}$ & 713 & 20,58 & 782 & 28,87 & S, DI \\
\hline & EAE (método simplificado) & \multicolumn{5}{|c|}{$648^{\circ} \mathrm{C} \quad 15,07$ mín } & \multicolumn{5}{|c|}{$648^{\circ} \mathrm{C} \quad 16,58 \mathrm{mín}$} \\
\hline \multirow[t]{3}{*}{ Capacidad limitada } & Pilar y base protegidos. Base empotrada. & 691 & 17,37 & 875 & 39,85 & Er, V & 731 & 22,37 & 875 & 40,57 & Er, V \\
\hline & & \multicolumn{5}{|c|}{ Tipo 2} & \multicolumn{5}{|c|}{ Tipo 3} \\
\hline & & \multicolumn{2}{|c|}{ STB } & \multicolumn{3}{|c|}{ Ruina } & \multicolumn{2}{|c|}{ STB } & \multicolumn{3}{|c|}{ Ruina } \\
\hline \multirow{3}{*}{ Empotradas } & Sin protección (SP) & 672 & 16,75 & 733 & 22,07 & $\mathrm{D}, \mathrm{DI}$ & 674 & 17,42 & 728 & 21,72 & S, DI \\
\hline & Pilar y base protegidos & 682 & 17,32 & 871 & 39,15 & Er, V & 683 & 17,93 & 866 & 38,38 & Er, V \\
\hline & EAE (método simplificado) & \multicolumn{5}{|c|}{$627^{\circ} \mathrm{C} \quad 14,65$ mín } & \multicolumn{5}{|c|}{$622^{\circ} \mathrm{C} \quad 14,97$ mín } \\
\hline
\end{tabular}



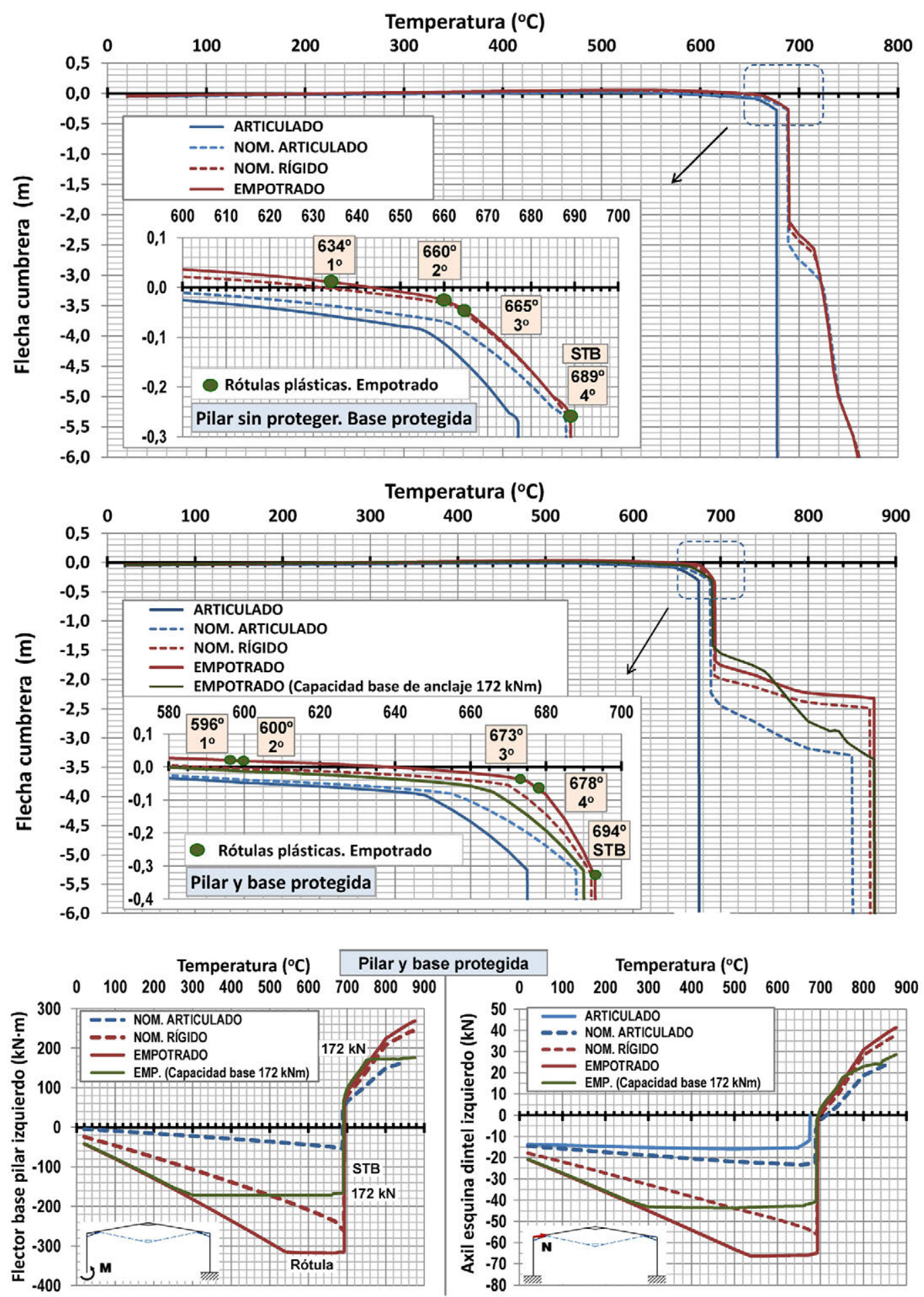

Figura 7. Pórtico tipo 1. Pilar sin proteger y base protegida: flecha vertical en cumbrera. Pilar y base protegida: flecha vertical en cumbrera, flector en la base del pilar y axil en esquina del dintel.

temperatura ambiente (Figura 7: $\mathrm{M}=172 \mathrm{kN}$ ), valor que como mínimo debe resistir la placa base y cimentación.

Para los casos de base nominalmente rígida y empotrada, si bien no se modifica ni la temperatura previa al STB ni la de ruina total, los desplomes crecen de forma más pronunciada. Se asemeja a los de la base nominalmente articulada aunque esté empotrada. En muchos casos, aunque exista una limitación del desplome, se pueden superar los 30' de resistencia al fuego.

- El nivel de carga es un parámetro importante de mayor influencia para cargas reducidas, en donde se aprecia que las curvas se separan más. Esto conduce a un aumento más que proporcional de la temperatura crítica en cualquier punto, pues son curvas a tramos equidistantes. Una aplicación es recurrir al sobredimensionado de la estructura para disminuir el grado de utilización. Los factores de carga indicados representan aproximadamente aumentar un perfil. Así, por ejemplo, si el factor de carga se reduce de 1 a 0,8 , se incrementa un $17 \%$ el tiempo resistido. Pero el incremento es del $47 \%$ respecto a la curva de factor o,6 (2 perfiles superior). Para pórticos con mayor carga de nieve, si en el dimensionado a temperatura ambiente es necesario subir 

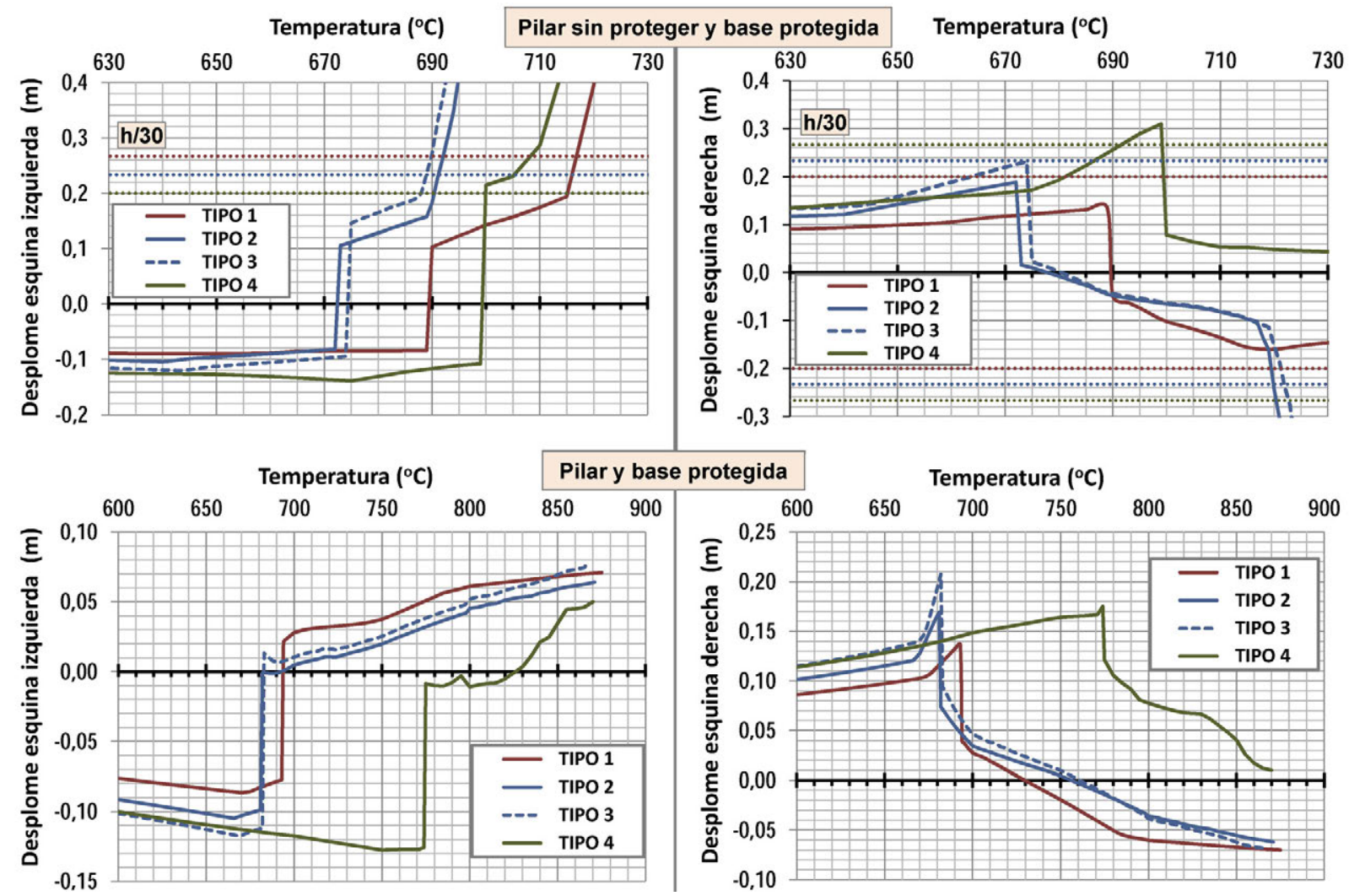

Figura 8. Desplome esquina izquierda y derecha de los pórticos analizados. Pilar sin proteger y base protegida, pilar y base protegida.

perfiles, ocurre una disminución neta del grado de utilización y la temperatura crítica es mayor.

- El incremento de longitud y de canto de los acartelamientos de esquina ejerce una influencia favorable aunque pequeña, salvo algún caso en el que si se desarrollan rótulas en cabeza de pilares, es indiferente.

\section{CONCLUSIONES}

- La metodología propuesta representa más fielmente la realidad y puede permitir justificar mayores tiempos de resistencia al fuego.

- Los pórticos de base articulada son indiferentes a la protección del pilar. Suelen colapsar hacia el exterior de la nave, lo que puede provocar daños y extender el fuego a edificios cercanos.

- Una pequeña rigidez en las bases de pilares puede ser suficiente para evitar la ruina hacia el exterior. Bases y cimentación deben dimensionarse para soportar el flector de vuelco. Una mayor rigidez y resistencia es favorable para disminuir los desplomes.
- Respecto a las bases empotradas o nominalmente rígidas, el momento resistido está limitado por la capacidad a flexión de la base y cimentación. Esta restricción altera muy poco las temperaturas del STB y ruina, pero el desplome puede ser elevado.

- La magnitud de las cargas ejerce una gran influencia en la temperatura crítica. Se aprecia más para cargas más bajas. Un ligero sobredimensionado asegura una resistencia al fuego R15.

- Si se protege total o parcialmente el pilar, éstos permanecen casi verticales y la estructura no afecta a la estabilidad de las fachadas, lo que es favorable. Los tiempos de resistencia al fuego aumentan de forma notable y sobrepasan los 30'.

- Mediante una estrategia adecuada de diseño de acartelamientos, bases y cimentación junto a un sobredimensionado no excesivo, se puede justificar una resistencia al fuego R3o como alternativa a la protección estructural con revestimientos. Para conseguir un R6o es necesario proteger la estructura, pero es posible realizar un buen ajuste.

\section{REFERENCIAS}

(1) Ministerio de Fomento (2011). Instrucción de Acero Estructural (EAE). España.

(2) Ministerio de Industria, Turismo y Comercio (2004). Real Decreto 2267/2004. Reglamento de seguridad contra incendios en los establecimientos industriales (RSCIEI). Boletín Oficial del Estado, $\mathrm{n}^{0} 303$. España.

(3) Simms, W. I., Newman, G. M. (2002). Single Storey Steel Framed Buildings in Fire Boundary Conditions. Reino Unido: The Steel Construction Institute (SCI). Publication P313.

(4) Wong, S. Y., Burgess, I. W., Plank, R. J. (2000). Simplified estimation of critical temperatures of portal frames in fire. Proceedings International Conference on Steel Structures of the 200os (paper 09.03). Istanbul.

(5) Wong, S. Y. (2001). The structural response of industrial portal frame structures in fire. Sheffield: University of Sheffield. 
(6) Vassart, O., Cajot, L. G., O’Connor, M., Shenkai, Y., Fraud, C., Zhao, B., De la Quintana, J., Martínez Aragón, J., Franssen, J. M., Gens, F. (2004, 5 de julio). 3D simulation of industrial hall in case of fire. Benchmark between ABAQUS, ANSYS and SAFIR. Interflam 2004. Proceedings of the 1oth international conference (pp. 1315-1324). London: Interscience Communications Limited.

(7) Bong, M. W. (2005). Structural Fire Performance of steel portal frame buildings. Canterbury: University of Canterbury.

(8) Song, Y. (2008). Analysis of industrial steel portal frames under fire conditions. Sheffield: University of Sheffield.

(9) Song, Y., Huang, Z., Burgess, I. W., Plank, R. J. (2009). The behavior of single-storey industrial steel frames in fire. Advanced Steel Construction, 5(3): 289-302.

(10) Rahman, M., Lim, J., Hamilton, R., Komlekci, T., Pritchard, D., Yixiang, X. (2011). Effect of column base strength on steel portal frames in fire. Structures and Buildings, 166(4): 197-216, doi: http://dx.doi.org/10.1680/stbu.11.00040.

(11) Moss, P. J., Dhakal, R. P., Bong, M. W., Buchanan, A. H. (2009). Design of steel portal frame buildings for fire safety. Journal of Constructional Steel Research, 65: 1216-1224, doi: http://dx.doi.org/10.1016/j.jcsr.2008.09.003.

(12) El-Heweity, M. M. (2012). Behaviour of portal frames of steel hollow sections exposed to fire. Alexandria Engineering Journal, 51: 95-107, doi: http://dx.doi.org/10.1016/j.aej.2012.06.004.

(13) Wilson, E. L. (2009). Integrated structural analysis and design software SAP2Ooo. Berkeley, California: Computers and Structures, Inc. http://www.csiamerica.com.

(14) Wen, Y. K. (1976). Method for random vibration of hysteretic systems. Journal of the Engineering Mechanics Division, ASCE, 102(EM2). 